\title{
Effects of Citric Acid and Desensitizing Agent Application on Non- fluorosed and Fluorosed Dentin: An In Vitro Sem Study
}

\author{
Mahajan Neha and Laxman K. Vandana*
}

Department of Periodontics, College of Dental Sciences, Davangere, Karnataka, India

\begin{abstract}
Fluorosis is one of the factors which bring about mineralisation changes in a dentinal structure leading to dentin. The purpose of the present study was to compare and evaluate the dentinal tubular changes in fluorosed and nonfluorosed teeth subsequent to the application of citric acid,strontium acetate based sodium fluoride (SAF) using scanning electron microscopy (SEM). Dentin specimens from healthy fluorosed and nonfluorosed teeth were included in the study. Each of them was grouped into acid treated and SAF treatment groups. Using SEM, the photomicrographs (3500x) of dentin specimens were evaluated. Results showed while there was a significant difference in tubular width of partial occlusion $\leq 25 \%$, being more in fluorosed group compared to nonfluorosed group after application SAF. Application of desensitising agents demonstrated higher number of dentinal tubular occlusion and diameter reduction in nonfluorosed dentin compared to fluorosed dentin. Summary: Root biomodification and desensitising agent procedure brings in definite difference between fluorosed and non-fluorosed dentin specimens.
\end{abstract}

Keywords: Citric acid, desensitising agent, fluorosed dentin, strontium acetate.

\section{INTRODUCTION}

For the causation of dentin hypersensitivity, dentinal tubular number, patency and diameter play important roles. Microscopic examination reveals that patent dentinal tubules more numerous and wider in hypersensitive dentine than in non-sensitive dentine [1]. These findings explain why some patients with cervical dentin exhibits dentin hypersensitivity and others donot. It has been reported that dentin hypersensitivity might be reduced physiologically by formation of intratubular crystals from the dentinal fluids and salivary minerals [2] or by the application of therapeutic chemical agents to occlude the dentinal tubules. Most of the compounds used in the treatment of dentinal hypersensitivity are thought to achieve therapeutic benefit by tubule occlusion [3].

The dentinal tubular occlusion can act both directly by blocking the tubules which reduces the fluid flow and indirectly on nerves by sealing off the tubules from the pain provoking stimuli. The most important variable affecting the fluid flow in dentin is the radius of the tubuli. Hence, measurement of tubule diameter gains importance for the assessment of effectiveness of desensitising agent. The difference in tubule diameter may be more important since fluid flow is proportional to radius by four times that is doubling the tubule diameter results in 16 fold increase in fluid flow thereby increasing hypersensitivity [4].

Dental fluorosis is a common complaint of subjects from Davangere district of Karnataka, India, which has naturally occurring high water fluoride levels. The dental fluorosis induced enamel hypomineralisation is well discussed [5].

\footnotetext{
*Address correspondence to this author at the Department of Periodontics, College of Dental Sciences Davangere, Karnataka, India;

Tel: +91-8192-30432 / 31285;

E-mail: vanrajs@gmail.com
}

Recently the dental fluorosis induced dentin changes have been reported. It has been observed that when the fluoride concentration in drinking water is $0.15-2.5 \mathrm{ppm}$, the mean plasma fluoride level has been seen to be $0.14-0.19 \mathrm{ppm}$ [6]. In a related study, a positive correlation between dentin fluoride concentration and dentin tubule size was shown, demonstrating wider dentinal tubules in teeth with higher level of fluoride concentration in dentin. It was also shown that high fluoride concentration of the tooth decreased the mineralisation rate in the tooth structure [7]. Fluoride concentration influenced crystal size [8] and also increased mineralisation time between matrix deposition and its mineralization [7]. Another possible hypothesis is that fluoride would influence crystal growth forming an impaired dental structure with wider dentinal tubules [8].

It has been reported that hypomineralised teeth have extreme sensitivity to cold and sweet stimuli and tooth brushing [9]. In support of these wider dentinal tubules and hypomineralisation, recently a Chinese study reported higher frequency $(9.26 \%)$ of tooth sensitivity compared to nonfluorosed subjects $(4.65 \%)$ [10]. Considering the wider dentinal tubules [8], hypo mineralisation effects there is a probability that dental fluorosed subjects would be more susceptible to dentine hypersensitivity. There is increased prevalence of dental fluorosis in and around Davngere district, Karnataka which led to the present study.

Various desensitising agents are available in the market which yielded promising results, but their therapeutic effects were relatively short-lived or decreased with time because these agents could be gradually removed by daily tooth brushing or acidic beverage drinking. In this study newly introduced strontium acetate fluoride has been used.

Medline search using keywords dentine hypersensitivity, desensitising agents, strontium acetate, fluorosed and non 
fluorosed teeth, scanning electron microscopy did not reveal any studies. There are no published papers addressing the treatment of dentine hypersensitivity in patient with fluorosis.

Currently, an initial attempt has been made in this scanning electron microscopic study to evaluate and compare the dentinal changes in healthy fluorosed and non fluorosed teeth after the application of Strontium acetate fluoride (SAF).

\section{MATERIALS AND METHODOLOGY}

A total of 6 fluorosed healthy and 6 non fluorosed healthy teeth were included in this study with age range of 20-40 years as peak incidence of dentin hypersensitivity is seen at this age. The freshly extracted teeth were obtained from the department of Oral and Maxillofacial Surgery, College of Dental Sciences, Davangere, Karnataka, India, and were used according to a protocol set forth by the research Ethics committee of Rajiv Gandhi University of Health Sciences, Karnataka, India.

The extracted teeth were required to meet the following inclusion criteria: fully erupted, extracted non-traumatically due to orthodontic reasons, no history of recent periodontal instrumentation or dental prophylaxis, for fluorosed teeth; the fluorotic enamel stains was confirmed by the clinical examination and history of the subjects hailing from natural high water fluoride areas in and around Davangere (fluoride concentration $>1.5 \mathrm{ppm}$ ). The exclusion criteria were: teeth with proximal caries extending to the cementum, fillings extending beyond cementoenamel junction (CEJ), and intrinsic stains caused by other reasons such as porphyria, erythroblastosis fetalis, tetracycline therapy, etc.

The extracted teeth were immediately washed in running tap water and stored in bottles containing $0.9 \%$ saline.

\section{Sectioning and Preparation of Dentin Specimens}

To prepare the discs, the enamel and cementum were removed with a high speed hand piece which was water cooled and sectioned with diamond saw.

Dentin discs of $2 \mathrm{~mm}$ thickness were prepared parallel to the CEJ with a low speed water cooled diamond saw and contra angled hand piece $1 \mathrm{~mm}$ and $2 \mathrm{~mm}$ below the CEJ using double sided diamond disk operated in a water cooled air motor.

\section{Treatment}

In this study $6 \%$ citric acid for a period of two minutes was applied for test group 1. Test group 2 was treated with strontium acetate fluoride desensitising agent. Dentin specimens were brushed with electric toothbrush for 2 minutes twice per day for 7 days, after which the dentin specimens were stored in artificial saliva for a period of 1 week [11].

\section{Preparation for SEM}

The specimens were placed in $2.5 \%$ Glutaraldehyde in 0.1 M Phosphate buffer ( $\mathrm{pH} 7.4$ ), for a minimum of 24 hours. Following washing and dehydration through a graded alcohol series (25\% to $100 \%)$, they were mounted on SEM stubs. Mounted specimens were air-dried for 48 hours and sputter coated with 30 to $40 \mathrm{~nm}$ of gold. Finally, specimens were examined by using a scanning electron microscope (JEOL-JSM-840A, operating at an accelerating voltage of $20 \mathrm{kV})$. Representative photomicrographs were obtained at 3500x for dentine surfaces.

SEM photomicrographs were assessed for following findings: (1) smear layer, (2) peritubular dentin, (3) number and diameter of tubules of different degrees of tubular occlusion.

Morphological parameters were given dichotomous scoring of present or absent. In the numerical data, counting of tubules was done manually using ADOBE PHOTOSHOP CS3 software.

The procedural steps used to count the completely occluded/partially occluded (CO/PO) dentinal tubular occlusion and the diameter measurements of the dentinal tubules were:

1) Nine square grids were used in each photomicrograph, so that the tubules could be measured in each grid.

2) Tubules present on the grid lines were included only in one of the grids to avoid repetition.

3) To overcome the difficulty to measure the tubular diameter in obliquely cut dentinal tubules, smallest area or diameter across the tubular orifice was taken to minimise the error caused by tubules cut obliquely [12].

4) The occlusion of tubules was considered as presence of a film which appeared as fibrous/amorphous over the dentinal tubular orifice.

In the current study a modified simple three point SEM categorical scale (0-3) was designed to analyse the degree of dentinal tubular occlusion after application of desensitising agents in SEM photomicrograph.

It was based on extent of dentinal tubule orifice closure

Type 0-Open dentinal tubules without occlusion i.e. acid treated specimens without desensitising agent application.

Type 1-Partial dentinal tubule occlusion $\leq 25 \%$ of dentinal tubule orifice (Fig. 1).

Type 2-Partial dentinal tubule occlusion of $>25 \%$ up to $75 \%$ of dentinal tubule orifice (Fig. 2).

Type 3- Nearly complete dentinal tubule occlusion of $>75 \%$ of dentinal tubule orifice (Fig. 3).

Diameter of dentinal tubules was measured using IMAGE J image analysis software (NIH Image, version 1.44, National Institutes of Health USA.

\section{STATISTICAL ANALYSIS}

Using the arbitrary numeric scale, a mean score and standard deviation were calculated for all specimens, at magnification of $3500 \mathrm{X}$. Non parametric test was used for intragroup and intergroup comparison (student unpaired t test). A p-value of 0.05 or less was considered for statistical significance. 


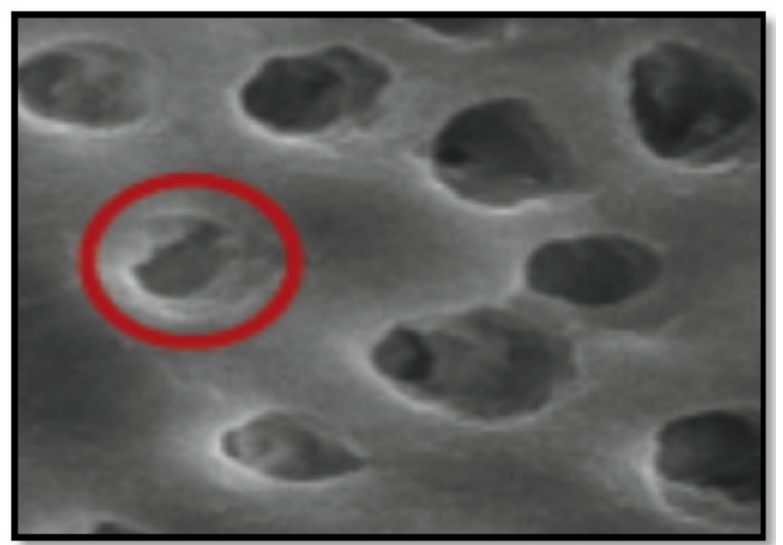

Fig. (1). Corresponds to Partial dentinal tubule occlusion of $\leq 25 \%$ of the dentinal tubular orifice

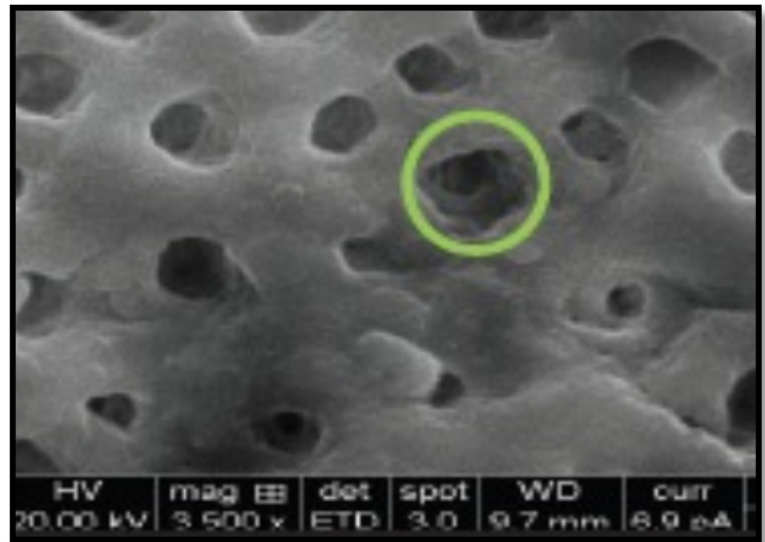

Fig. (2). Corresponds to 'Partial dentinal tubule occlusion of $>25 \%$ up to $75 \%$ of the dentinal tubular orifice.

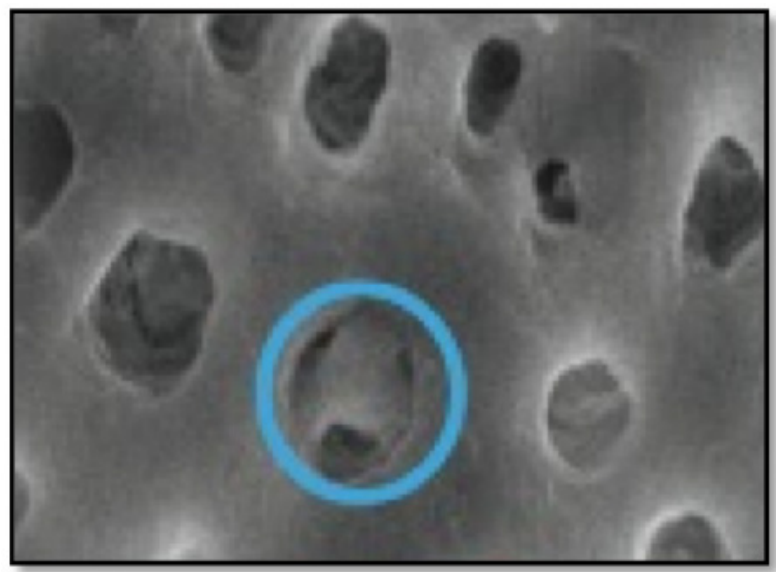

Fig. (3). Corresponds to Partial dentinal tubule occlusion of $>75 \%$ of the dentinal tubular orifice.

\section{RESULTS}

A total of 46 periodontally healthy dentin specimens from both fluorosed $(n=23)$ and nonfluorosed $(n=23)$ teeth were included in this in-vitro study to evaluate the effect of acid etched dentin specimens treated with SAF (strontium acetate fluoride desensitising agent.) The results of the study are interpreted in Graphs 1and 2.
The number of completely occluded (Type 3 ) and partially occluded $>25 \%$ (Type 2 ) dentinal tubules was significantly higher in nonfluorosed as compared to the fluorosed group (Graph 1).

The fluorosed dentin demonstrated significantly wider $(0.64 \mu \mathrm{m}$ and $0.64 \mu \mathrm{m})$ dentinal tubular diameter than the nonfluorosed dentin $(0.32 \mu \mathrm{m}$ and $0.62 \mu \mathrm{m})$ of partially occluded $\leq 25 \%$ and $>25 \%$ upto $75 \%$ (Type 1 and Type 2 ) respectively (Graph 2).

\section{DISCUSSION}

A large number of treatment options are available for managing dentinal hypersensitivity but there is currently no "gold standard" treatment option for dentin hypersensitivity.

The desensitising agents used in this is strontium acetate based sodium fluoride (Sendoyne, Sarvotham Care Limited, Baddi, Himachal Pradesh, India) have been used.

The higher occurrence of dentin hypersensitivity in fluorosed teeth [10], wider dentinal tubule and impaired dentinal tubular structure in healthy fluorosed dentin [7] provided the basis for this study. The objective of the study was to compare the in vitro efficacy of the desensitising agents on dentin discs of healthy fluorosed and nonfluorosed dentin which were acid etched to simulate hypersensitive dentin that is characterised by wider dentinal tubular orifice.

Ideally the number of completely occluded tubules should be higher than the partially occluded tubules. However, the graded dentinal tubular occlusion in terms of complete occlusion of $>75 \%$, partial occlusion of $>25-75 \%$ and $\leq 25 \%$ evaluates the efficacy of the desensitising agent objectively using dependable and reproducible computer methods, hence providing an ideal measure of desensitising effect of comparable agents. As per author's literature search, this study is the first of its kind to introduce it.

The results of this study are discussed as follows

The number of completely occluded (Type 3 ) and partially occluded $>25 \%$ (Type 2 ) dentinal tubules was significantly higher in nonfluorosed as compared to the fluorosed group. No literature exists to compare the efficacy of strontium acetate fluoride in fluorosed and nonfluorosed group (Graph 1).

Dentinal tubular diameter affects the fluid flow and the measurement of the same is the proper evidence of desensitising agent effectiveness clinically.

The clinical implication of dentinal tubular occlusion measure may be considered as a direct measure of efficacy of the desensitising agent in dentinal tubular occlusion. Various degrees of dentinal tubular occlusion diameter could provide a guideline for the manufacture of desensitising agent either trying to adjust its concentration, frequency and number of clinical applications so as to enhance the number and higher degree of dentinal tubular occlusion which is known to decrease the sign and symptom of dentinal hypersensitivity.The dentinal tubular diameter measurement has been attempted for the first time in the literature as per the medline search. The diameters of tubules with partially dentinal tubular occlusion of Type $2(>25 \%)$ and Type $1(\leq 25 \%)$ were measured using ImageJ software. 


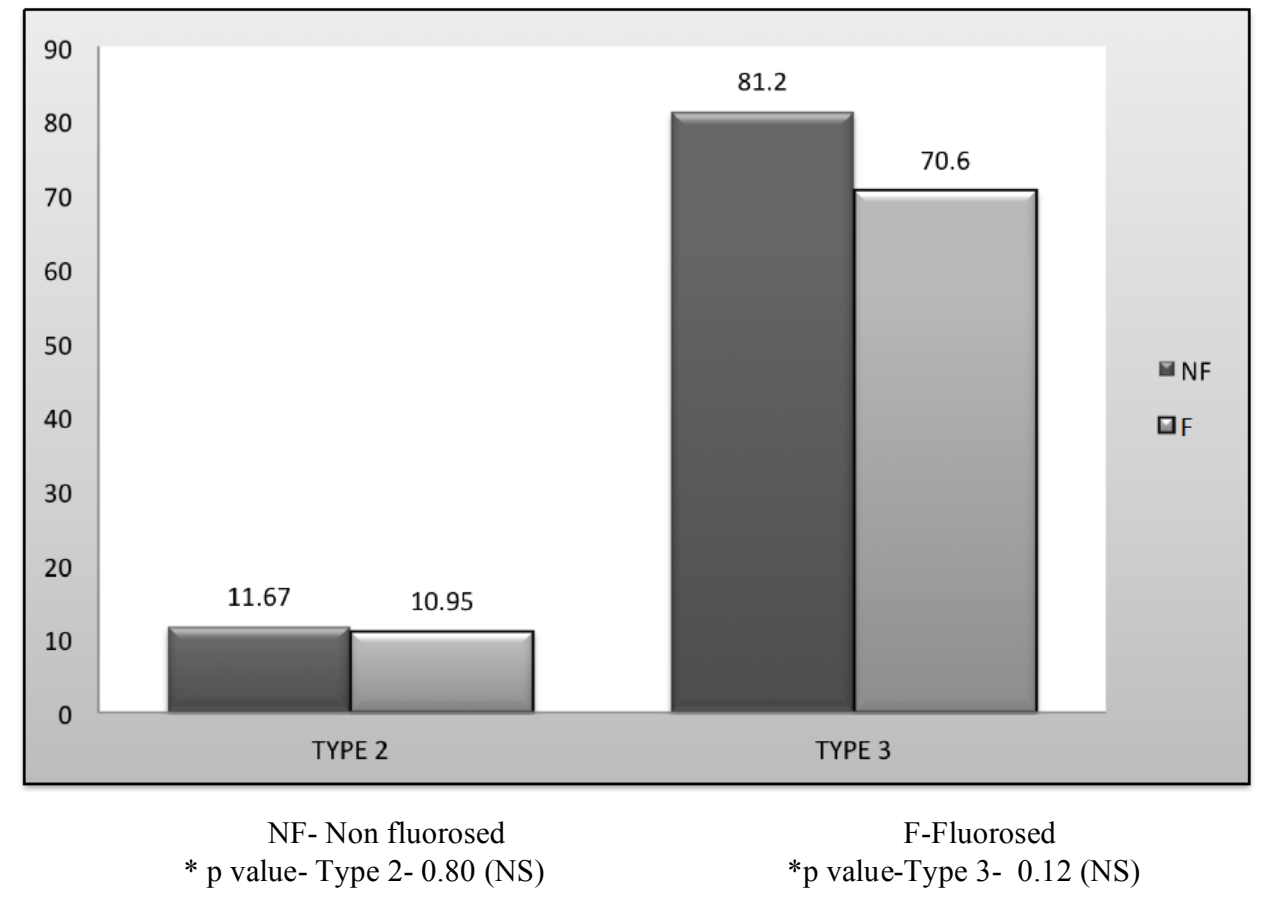

Graph 1. Comparison of number of completely occluded and partially occluded dentinal tubules $>25 \%$ in non fluorosed \& fluorosed dentin treated with SAF.

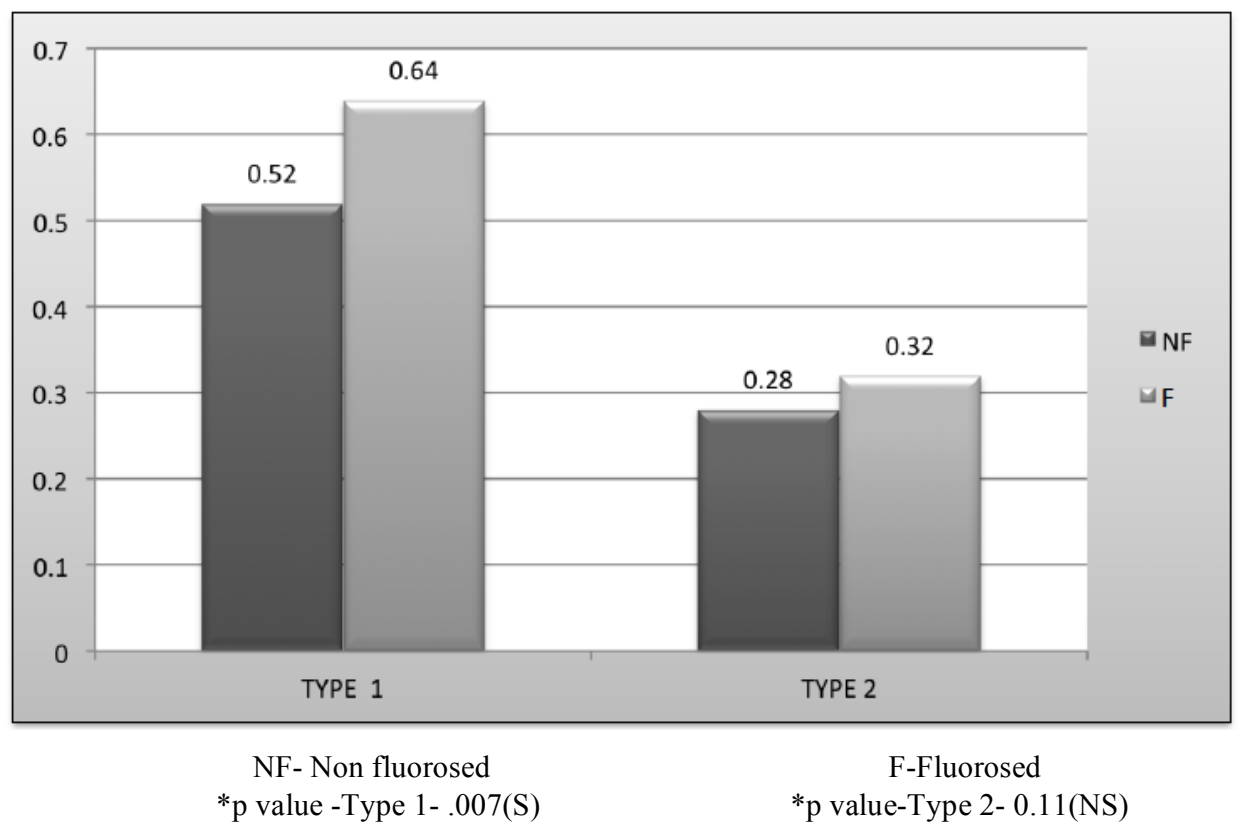

Graph 2. Comparison of Mean diameter $(\mu \mathrm{m})$ of partially occluded tubules $(>25 \%$ and $\leq 25 \%)$ between nonfluorosed \& fluorosed dentin with SAF.

The significantly higher tubular diameter in fluorosed $(1.62 \pm 0.32 \mu \mathrm{m})$ and nonfluorosed $(1.33 \pm 0.49 \mu \mathrm{m})$ dentin specimens could be attributed to the higher levels of fluoride in fluorotic dentin. Fluoride concentration has been shown to influence crystal size, and same evidence indicates that fluoride has an effect on cell function, either directly through interactions with the developing ameloblasts and/or odontoblasts or more indirectly by interacting with extracellular matrix. This action would then explain the positive relationship between dentin tubule size and fluorosed tooth, where hypomineralizaton caused by fluoride concentration, would create wider dentinal tubules. Another hypothesis is that fluoride would influence crystal growth, forming an impaired dental structure [8] with wider dentinal tubules.

Sample size distribution was different with various studies ranging from sample size 2 to 20 [13-15] as compared to present study sample size of 46. Perhaps one of the major problems in testing for hypersensitivity is that the condition shows no physical signs. Therefore it is not surprising that considerable interest has been shown towards in vitro 
evaluation of both the condition of hypersensitivity and the treatments involved in its management. Surprisingly, there is minimum literature available to compare the results of present study. The use of both control and test dentin specimens from same tooth has provided minimum bias conducting this study.

However, the percentage difference amongst the number of dentinal tubule occlusion and their diameter reduction provided the important information on efficacy of SAF, i.e statistical significance is not equal to in vitro efficacy/ clinical importance. It only tells us that the observed differences in the outcome are unlikely to be due to "chance alone." This may be a useful first step in evaluating the importance of the observed differences, but it is important to examine the in vitro results of the observed differences from clinical view. It should clearly be understood that a difference which is statistically significant may or may not be clinically relevant and a difference which is statistically non-significant, does not necessarily imply a clinically unimportant finding [16].

SAF has shown higher values for number of dentinal tubular occlusion and dentinal tubular diameter reduction in nonfluorosed group, suggestive of better dentinal tubule occluding effect and the translation of this data clinically suggests a better desensitising agent in nonfluorosed group than fluorosed group. Owing to wider dentinal tubules in fluorosed group and the results of present study is suggestive of lesser effectiveness of SAF in fluorosed group, it appears that there is a need to evaluate the different concentrations of arginine (ARG), duration and frequency of its application to treat hypersensitivity in fluorosis dentin, using SEM followed by clinical evaluation.

\section{Future Studies}

Since fluorosis has global occurrence, studies have to be conducted from those areas of endemic fluorosis and manufacture of desensitising agents suitable to fluorosed dentin is recommended. The depth of penetration of desensitising agent is required to be studied. Also changing the concentration of the desensitising agents could help to improve the treatment effect for dentin hypersensitivity in fluorosed group keeping in mind the hypo mineralised fluorosed dentin. Further studies need to be conducted to assess the required concentration, duration and frequency of the desensitising agent application for hypersensitive fluorosis teeth.

\section{CONCLUSION}

Since the dentin tubule diameter differs between fluorosed and non fluorosed teeth(), the application of desensitising agents demonstrates different degrees of dentinal tubular occlusion as interpreted by SEM evaluation. The clinical transfer of the study is to primarily make aware.
The basic structural difference known globally so that the treatment of fluorosed dentin would require alteration in desensitising formulation so that its application will lead to effective dentinal tubular occlusion. The effective concentration of desensitising agent requires to be determined in further studies.

\section{CONFLICT OF INTEREST}

The authors confirm that this article content has no conflict of interest.

\section{ACKNOWLEDGEMENTS}

Declared none.

\section{REFERENCES}

[1] Louis H. Burman dentinal sensation and hypersensitivity: a review of mechanism and treatment alternatives. J Periodontol 1984; 45(4): 216-21

[2] Yoshima M, Noiri Y, Ozaki K, uchida A, Ischikava Y, Ishida H. Transmission electron microscopic characterisation of hypersensitive human radicular dentin. J Dent Res 1990; 69(6): 1293-7.

[3] Absi EG, Addy M, Adams D. Dentine hypersensitivity; a study of patency of dentinal tubules in sensitive and nnon sensitive cervical dentine. J Clin Periodontol 1987; 14: 28-32.

[4] Addy MB. Dentin hypersensitivity: new perpective on an old problem. Int Dental J 2002; 52: 367-75.

[5] Aoba T. The effect of fluoride on apatite structure and growth. Crit Rev Oral Biol Med 1997; 8: 136-53.

[6] Singer L. Amstrong WD. Regulation of human plasma fluoride concentration. J App Physiology 1960; 15: 508-10.

[7] Anya PGF, Viera RH, Mircea D, Limeblack H, Marc D. Grynpas. Fluoride's effect on human dentin ultrasound velocity (elastic modulus) and tubule size. Eur J Oral Sci 2006; 114: 83-8.

[8] Vieira APGF, Hancock R, Schwartz M, Limeback H, Grynpas MD. How does fluoride concentration in tooth structure affects apatite crystal? J Dent Res 2003; 82: 909-13.

[9] Jälevik B, Klingberg GA. Dental treatment, dental fear, and behavior management problems in children with severe enamel hypomineralization of their first permanent molars. Int J Pediatr Dent 2002; 12: 24-32.

[10] Mine OT,Yener O, Tugba S, Yonca S, Yesim Kirziogolu F. Tooth sensitivity in fluorotic teeth. Eur J Dent 2011; 5(3): 273-80.

[11] Arrais CAG, Micheloni CD, Giannini M, Daniel CN. Occluding effect of dentifrices on dentinal tubules. J Dent 2003; 31: 577-84.

[12] Schilke R, Lisson JA, Baub, Geurtsen W. Comparison of the number and diameter of dentinal tubules in human and bovine dentine by scanning electron microscopic investigation. Arch Oral Biol 2000; 45: 355-61.

[13] Mordan NJ, Barber PM, Gillam DG. The dentine disc: a review of its applicability as a model for the in vitro testing of dentine hypersensitivity. J Oral Rehabilit 1997; 24: 148-56.

[14] Gillam DG, Khan N, Mordan NJ, Barber PM. Scanning electron microscopy (SEM) investigation of selected desensitizing agents in the dentine disc model. Endod Dent Traumatol 1999; 198-204.

[15] Pashley DH, Kalathoor S, Burnham D. The effects of calcium hydroxide on dentin permeability. J Dent Res 1986; 65(3): 417-20.

[16] Pihlstrom BL, Curran AE, Voelker HT, Kingman A. Randomized controlled trials: what are they and who needs them? Periodontology 2000 2012; 59: 14-31.

\footnotetext{
Received: December 13, 2014

Revised: January 10, 2015

Accepted: January 20, 2015

(C) Neha and Vandana; Licensee Bentham Open.

This is an open access article licensed under the terms of the Creative Commons Attribution Non-Commercial License (http://creativecommons.org/licenses/by-nc/3.0/) which permits unrestricted, non-commercial use, distribution and reproduction in any medium, provided the work is properly cited.
} 